\title{
La dialéctica de la pregunta y la respuesta como estrategia de autorregulación en la construcción de un aula virtual inteligente
}

\author{
The Dialectics for the Question and the \\ Answer, a Strategy of Autoregulation for the \\ Constructions of an Intelligent Virtual Room
}

Francisco Alonso Chica Cañas*

Fecha de recepción: 23 de enero de 2009

Fecha de aprobación: 27 de febrero de 2009

\section{Resumen}

El aula virtual inteligente con enfoque problematizador sustentado en la dialéctica de la pregunta y la respuesta es determinante en la construcción de una pedagogía, en el sentido de poner al descubierto los cuestionamientos del sí y del no, tanto del argumento como del contraargumento. Por tanto, una conversación auténtica en el aula virtual implica potenciar todos los sentidos para ir en búsqueda de la verdad, a partir de la precomprensión de la pregunta para convertirse en una persona intolerante, porque siempre intenta demostrar la verdad en medio de la incertidumbre y la certeza del conocimiento. Entonces, el aula virtual inteligente debe establecer una pretensión de validez del aprendizaje con base en las experiencias previas del

Decano de la Facultad de Educación, Vicerrectoria de Universidad Abierta y a Distancia, Universidad Santo Tomás. Correo electrónico: franciscochica@ustadistancia.edu.co. 
mundo cotidiano y del mundo de la vida, tomando el presente, el pasado y el futuro del conocimiento, con el fin de problematizar el conocimiento referenciado en la propia existencia de la comunidad educativa virtual.

La universidad de educación superior abierta y a distancia debe asumir el reto de las aulas virtuales inteligentes. Esto implica replantear el horizonte y la concepción de la educación en línea con base en una concepción antropológica del ciberespacio, entendiendo que la identidad cultural forma parte del mundo globalizado y de la localización del individuo. En otras palabras, las aulas virtuales inteligentes se caracterizarán por una formación integral que tiene en cuenta los símbolos, el lenguaje, los signos y la cultura en el proceso de aprendizaje del estudiante. Además, de apropiarse la tecnología de punta para un conocimiento de la vista, el oído, el tacto y lenguaje virtual, favoreciendo un conocimiento con identidad humanista, con el fin de responder a los cuestionamientos sustentados en la razón, en el lenguaje y la verdad.

Palabras clave: aula virtual inteligente, el ser ahí, la acción comunicativa, la pregunta y la respuesta, el lenguaje, la razón y la verdad.

\section{Abstract}

The intelligent virtual room within a problematization approach sustained in the dialectics of the question and the answer is a determiner for the pedagogy construction, since it reveals the questions of yes and no, for both the argument and the counter-argument. Therefore, an authentic conversation in a virtual room implies to promote all the senses to go in search of the truth from the pre-comprehension of the question to turn into an intolerant person, since it is always demonstrated the truth in the middle of the uncertainty and the certainty of knowledge. Then, the intelligent virtual room must establish a pretension of validity of the learning according to previous experiences of the daily world and of the world of the life, taking the present, the past and the future of knowledge, to problematize knowledge referred in the own existence of the educational virtual community.

Higher Distance Education must assume the challenge of intelligent virtual rooms. This implies restating the horizon and the conception of online education with an anthropologic conception of the cyberspace, understanding that the cultural identity makes part of the globalized world, as well as the location of the individual. In other 
words, the virtual intelligent rooms will be characterized by an integral formation taking into account the symbols, the language, the signs, and the culture in the learning process of students. In addition, of the appropriation of technology for the knowledge of the sense of sight, the sense of hearing, the sense of touch and the virtual language, in favor of knowledge with a human identity to answer questions sustained in the reason, the language and the truth.

Key words: intelligent virtual room- "being there"- communicative action- the question and the answer- language- reason and the truth.

\section{Introducción}

El aula virtual inteligente encontrará en la sociedad, en el lenguaje, en la cultura, en el mundo cotidiano y en el mundo de la vida un horizonte de posibilidades para que el aprendiente crezca en la inteligencia emotiva, afectiva, social, cultural y científica. Por tanto, la pregunta y la respuesta en el aula virtual inteligente se constituye en un laboratorio pedagógico para la resignificación del conocimiento, tomando como punto de partida la propia existencia del ser ahí arrojado en el mundo. El hecho de interactuar con otros en el aula virtual inteligente crea temor a la crítica por parte del docente y de los compañeros, al encontrarse en situaciones desconocidas que requieran de certeza del conocimiento en medio de la incertidumbre.

Entonces, en medio de la pregunta y la respuesta el aprendiente debe obtener respuesta sobre sí mismo, sobre la sociedad y el mundo a través de la indagación, la exploración, el argumento y el contraargumento, con el fin de construir conceptos y teorías con base en la comprensión y en interpretación de indeterminaciones dialécticas temáticas para construir temas problematizadores del conocimiento. De ahí que el aula virtual inteligente deba estar acorde con un modelo pedagógico, un modelo evaluativo, un modelo de las prácticas pedagógicas y un modelo curricular que problematice el conocimiento a partir de las proposiciones del aprendiente, para así aprender a ver, juzgar y actuar con la colaboración de los demás participantes del aula virtual inteligente.

El ciberespacio es una gran oportunidad para el aprendiente para compartir las experiencias del ser ahí arrojado en el mundo, lo cual implica dominar un lenguaje y una simbología digital, con el propósito de producir proposiciones que correspondan con las representaciones ideales y reales de la dialéctica de la pregunta y la respuesta. Por otra parte, el lenguaje en el aula virtual inteligente es clave porque instaura un diálogo existenciario, al apoyarse en la vista y oídos de los demás, lo cual proporciona una problematización del conocimiento sensorial, racional y experimental, con el interés de validar los postulados del pensamiento hipotético.

El aula virtual inteligente tendrá que caracterizarse por responder a la pregunta acerca del 
lenguaje, la razón y la verdad. En otros términos, ¿cuál es el lenguaje, la razón y la verdad de la antropología del ciberespacio?; ¿cuál es la razón, el lenguaje y la verdad sobre los símbolos, los signos en torno a la creencia y costumbres de los grupos sociales?; ¿cuál es el lenguaje, la razón y la verdad de la ciencia y la tecnología con una visión humanista? Es importante que el estudiante comprenda la tecnología como un medio de interacción virtual para promover a la otra persona dentro del proyecto de vida personal. Es decir, cuando se habla de aula virtual inteligente es porque tiene la capacidad de abordar la problemática de una antropología del ciberespacio.

\section{El ser ahí como referente para la construcción del aula virtual inteligente}

El aula virtual inteligente tendrá como referente la cultura, la sociedad y el lenguaje; es un espacio en el cual el aprendiente interactúa en el ámbito del mundo cotidiano y el mundo de la vida. Además, la pregunta y la respuesta del aula virtual inteligente parten de la inteligencia afectiva, social, emotiva, comunicativa y estado de autoestima, como también de la existencia del aprendiente. En otras palabras, el horizonte de la respuesta se nutre de la experiencia existencial y vital del aprendiente y del colectivo con el cual interactúa, creando un mundo de posibilidades de acuerdo con la inteligencia sentimental y estado de ánimo.

Por tanto, la inteligencia del aula virtual inteligente se encuentra en relación directa con la experiencia existencial del aprendiente, determinando una visión de mundo situacional. Esto significa que la respuesta a la pregunta tiene como origen la vivencia del aprendiente, por lo cual se constituye en un gran laboratorio para abrirse a múltiples situaciones que proporciona el mundo de la vida. Es decir, la inserción del individuo en la sociedad proporciona un referente valioso para que el aprendiente se encuentre con la propia existencia de sí mismo. Por consiguiente, la pregunta y la respuesta involucran una relación de sentidos con la vida misma y el entorno, conllevando a resignificar el conocimiento con base en la experiencia existencial. Esto quiere decir, que la dialéctica de la pregunta y la respuesta tendrá sentido cuando el individuo es "uno con los otros", creando un escenario de posibilidades para comprender e interpretar al hombre, el mundo y la vida en una experiencia virtual inteligente de aprender a compartir la existencia con los otros.

El aprendiente, cuando plantea la pregunta y la respuesta en el aula virtual inteligente, se enfrenta a algo desconocido, a algo que genera temor, en cuanto es amenazado por ir en la búsqueda de la respuesta certera en medio de la incertidumbre. De ahí que la persona se sienta temerosa, no por la interacción con los demás, sino por encontrarse en una situación que puede herirlo, por no comprender la razón del conocimiento, por refutarle y contra argumentarle, por no compartir la visión del mundo de la vida, cuando, al intentar dar la mejor respuesta, es criticada por los compañeros y el docente, cuando no encuentra la utilidad del conocimiento en el mundo cotidiano y en el mundo de la vida, desconoce todo el desarrollo 
de las Tecnologías de la Información y la Comunicación, aprende por un interés inmediato. Entonces, el temor se convierte en pavor, al encontrarse con situaciones desconocidas para el "ser ahí" del aprendiente.

El aprendiente tiene que comprender y explicar el conocimiento. Por tal motivo, consiste en hacer frente para llegar a ser en el mundo. En otros términos, comprender la respuesta de la pregunta implica poder ser para alcanzar una respuesta satisfactoria sobre sí mismo, sobre la sociedad y el conocimiento objetivo. Comprender es alcanzar diferentes modos de estrategias para desarrollar una metainteligencia en lo sociocultural, lo intelectual, lo ético, lo disciplinar, la bioética y otros. En consecuencia, "el carácter de proyección del comprender quiere decir, además, que el comprender no aprehende temáticamente aquello mismo sobre lo que proyecta, las posibilidades" (Heidegger, 2007a). La comprensión se entiende como las posibilidades de indagar, de explorar, de cuestionar, de explicar, de argumentar y contra argumentar, con el fin de validar el pensamiento hipotético en función del bien común. Comprender para la vida y la vida profesional; comprender con los sentidos, comprender como un ser íntegro abierto al mundo.

Además de comprender, se debe interpretar la información y los contenidos expuestos en el aula virtual inteligente. Esto quiere decir que los contenidos son una excusa para que el aprendiente descubra el significado según la aprehensión y la construcción de conceptos que desemboquen en la formulación de teorías. Por tanto, "la interpretación de algo como algo tiene sus esenciales fundamentos en el 'tener', el 'ver' y el 'concebir previos'. Una interpretación jamás es una aprehensión de algo dado llevado a cabo sin supuesto" (Heidegger, 2007b).

Comprender e interpretar parten de las competencias, de las habilidades, de las destrezas y de las actitudes adquiridas por el aprendiente en el mundo cotidiano y en el mundo de la vida; existe una decantación de las indeterminaciones para convertirlas en temas certeros de logros que adquiridos con anterioridad. Sin embargo, lo que el aprendiente posee por circunstancias de la experiencia de la vida y de la existencia es el resultado del contacto de los sentidos con la realidad circundante, del cual pasa de los preconceptos a los conceptos.

El aula virtual inteligente tiene significado para el aprendiente, para la comunidad académica y la comunidad local o regional, cuando parte de la experiencia previa del "ser ahí"; asimismo, es tener en cuenta las emociones, los sentimientos, los afectos, los razonamientos previos y la concepción que tengan de la vida, para contextualizar el conocimiento social y el conocimiento objetivo con la existencia y vitalismo del aprendiente. El aula virtual inteligente induce al cambio y a la transformación del modelo pedagógico, del modelo evaluativo, de las prácticas, de la concepción del enfoque curricular, de la manera de representar y problematizar el conocimiento por problemas -más que por cumplir con unos contenidos contemplados por el estado o por una institución-, el uso adecuado de las nuevas tecnologías con una concepción pedagógica problematizadora y la concientización de implementar un nuevo 
juego de roles entre docente y aprendiente. El significado del aula virtual inteligente radica en comprender e interpretar la pregunta y la respuesta desde la experiencia intersubjetiva del individuo enmarcado en el contexto de lectura y relectura de la sociedad y la sociedad del conocimiento; a la vez, que el currículo debe ser innovador y significativo para la comunidad académica y la institución como tal.

Las proposiciones del aula virtual inteligente parten de los intereses de los aprendientes, quienes buscan, a través de los planes de estudio, la evaluación, la investigación, la proyección social, una excusa para problematizar el conocimiento, de acuerdo con el sentido que el estudiante encuentre en la acción de interactuar consigo mismo y con los demás, la acción de relacionar el conocimiento de la globalización, y la acción de comprender e interpretar el conocimiento nativo con el conocimiento científico. Entonces, el aula virtual inteligente persigue la verdad, lo cual implica comprender el significado de la pregunta y la respuesta para ir en búsqueda de la verdad del conocimiento científico, humano, ético, social y sociocultural. En consecuencia, la primera proposición consiste en que el aprendiente comprenda, analice e interprete con sentido lo que comprende por sí mismo. La segunda proposición radica en la comprensión del entorno, el cual se constituye en el significado del predicado. La tercera proposición significa la comunicación, es decir, aprender a ver, a juzgar y actuar con la participación del otro.

La proposición en el aula virtual inteligente tiene que ver con los juicios que emite el aprendiente, los cuales tienen valor por buscar la validez y la objetividad, al partir del referente de la propia existencia, de la realidad sociocultural, de la problemática política y de la influencia de la sociedad del conocimiento. El hecho de formular una proposición toca al ser ahí, un ser ahí arrojado en el mundo, un ser ahí inmerso en el mundo circundante y en el mundo del ciberespacio. Con base en este último cambio la proposición en el aula virtual inteligente concibe el escenario del ser ahí mediada por una máquina que pone en contacto al aprendiente con una realidad virtual que le brinda la oportunidad de interactuar con una comunidad digital que trata de aprender una simbología, una semiótica, un lenguaje en el cual todos se encuentran al mismo tiempo y tienen la oportunidad de producir proposiciones que corresponden a la realidad del ciberespacio. De ahí que:

[...] la tecnología, el dinero y el ciberespacio hacen del hombre un cazador, un propietario, un dominador más aterrador que nunca, pero los grandes objetos contemporáneos sólo le confiere estos poderes forzándole a vivir la experiencia propiamente humana de la renuncia a la presa, de la deserción del poder y del abandono de la propiedad. La experiencia de la virtualización (Lévy, 1999).

El aprendiente en el aula virtual inteligente tiene que habérselas con proposiciones que emergen de las sensaciones, de los sentimientos, de los afectos, de las emociones, de la confrontación con el mundo y el ciberespacio, del conocimiento del entorno y de la sociedad, de la política, de la economía, de la cultura, de la violencia y la muerte que vive Colombia, de la voracidad del neoliberalismo y la glo- 
balización del capitalismo versus la pobreza, con un conocimiento especializado y con los preconceptos del conocimiento vulgar. Por ende, las proposiciones tienen que ver con las representaciones ideales que deben pasar el umbral de las representaciones reales, con el fin de construir un conocimiento con sentido que conlleve a la producción de nuevos conceptos y teorías, resultado de una comprensión de sintesis. En otros términos, unir y separar las proposiciones, a partir de ver, juzgar y actuar, induce a una comprensión hermenéutica de un ser ahí que intenta responder la dialéctica de la pregunta y la respuesta abierto al mundo del humanismo, la ciencia y la tecnología.

\section{El ser ahí del aprendiente en el contexto del lenguaje y la acción comunicativa del aula virtual inteligente}

El aprendiente se encuentra abierto al mundo para establecer un diálogo de comprensión acerca de todo lo que lo rodea, siendo el habla el medio para expresar las significaciones del pensamiento, constituyéndose las palabras en el repertorio que proporciona significaciones. La palabra tiene la característica de partir de la experiencia mundana del aprendiente, de los preconceptos, de los imaginarios que tenga de la realidad circundante, de las experiencias referidas a la existencia. Entonces, el ser ahí del aprendiente le permite hablar de la existenciariedad, en cuanto aprende a comprender el mundo con la mirada y los actos de habla de los demás, entablando un diálogo sobre diversos intereses que atañen al mundo cotidiano y al mundo de la vida. El habla contribuye a mantener al aprendiente abierto al mundo, constituyéndose en un acto comunicativo existenciario, llegando a crecer con el otro para formar parte de la sociedad del conocimiento, al tiempo que busca lograr un reconocimiento social con base en el dominio de una disciplina.

El aprendiente, como un ser abierto al mundo, trata de encontrarse y comprender a los demás, con el fin de entablar un diálogo; de hecho, con todos, tomando como base el oido y la vista. De ahí que el conocimiento del aprendiente es sensorial, lo cual posibilita la formación de representaciones mentales acorde con la experiencia existenciaria. Por esta razón, la problematización del conocimiento en el aula virtual inteligente se fundamenta en la acción comunicativa, la cual adquiere el estatus de habla y contrahabla, la cual aporta los sentimientos, los afectos, los intereses, las pasiones, las emociones, las metas, en fin, toda la realidad existencial del hombre. El aprendiente llega a la mayoría de edad cuando logra entender y comprender las cosas por sí mismo, generando nuevos conceptos y teorías producto de la articulación del lenguaje, el entorno y la existencia como tal. Por consiguiente, la problematización del conocimiento en el aula virtual inteligente se logra por estar abierto al mundo, con el fin de gestar nuevos actos de habla acorde con una metainteligencia hipotética, que vuelve a problematizar el conocimiento y producir nuevos conceptos con base en los conocimientos previos.

El aprendiente encuentra en el lenguaje un medio ideal de representación de la realidad, 
con el fin de compartir el conocimiento y las experiencias de lo cotidiano, del mundo de la vida y del conocimiento como tal. Por tal razón, "la conversación a través de la que los hablantes se entienden sobre aquello que pasa en el mundo y sobre aquello que les pasa a ellos mismos es para Habermas el modelo paradigmático del uso del lenguaje" (Fabra, 2008). Sin embargo, la acción comunicativa se debe ubicar en dos planos de la pragmática del acto de habla: primero que el estudiante logre una comprensión de la utilidad del conocimiento con respecto al contexto y al mundo de la vida -visto desde la perspectiva de Gadamer-. Segundo que dimensione la problematización de la acción comunicativa en el aula virtual inteligente a partir de la interacción con otras personas, con el propósito de dialogar en medio del discenso/consenso para lograr avances sobre temas cognitivos, metacognitivos, éticos y morales, contextuales, científicos y experimentales, afectivos y emotivos, etc. En otros términos, la problematización del aprendizaje no se centra únicamente en la comprensión del conocimiento, también requiere formarse en la prudencia, la criticidad, el diálogo, la tolerancia, la solidaridad, la caridad y el respeto por el otro. Es decir, aprender a comunicarse con la otra persona para ser buenas personas antes que formarse en seres altamente competitivos para responderle a una sociedad global cientifica y tecnológica.

La problematización del aprendizaje entre docentes y estudiantes en el aula virtual in- teligente, conlleva a la pretensión de validez argumentativa en cuanto lleguen a un entendimiento en medio de la diferencia, con el interés de coordinar acciones sobre nuevas problematizaciones y nuevos retos de comprensión de significado, tanto a nivel del conocimiento intersubjetivo, conocimiento social y conocimiento objetivo. Entonces, oponente y proponente se deben comunicar correctamente (semántico y sintáctico) y presentar las proposiciones en forma aceptable. En otras palabras, las frases u oraciones que emiten los interlocutores son susceptibles de problematización y falsación, cuando persigue la búsqueda de la verdad en medio de los valores y los antivalores, en medio de un afán capitalista y depredador, en medio de recrear el conocimiento de la sociedad global con intereses netamente pragmáticos, en medio de la tensión de la violencia y los conflictos, en medio del dominio la ciencia y la tecnología con fines lucrativos. Esto implica realizar una interpretación recíproca de índole cognitivo y moral, que rescate en primera instancia a la persona y a la sociedad, además de incentivar la creatividad con intereses de apropiación de conceptos y teorías. En consecuencia, la función significativa consistiría en: "1) entenderse, 2) con otro, 3) sobre algo” (Fabra, 2008). Es decir, el significado de los actos de habla verbal y no verbal parte de la experiencia del reconocimiento de la otredad antes de asumir una comprensión de lo cognitivo. 


\section{La pregunta y la respuesta desde la perspectiva del lenguaje, la razón y la verdad}

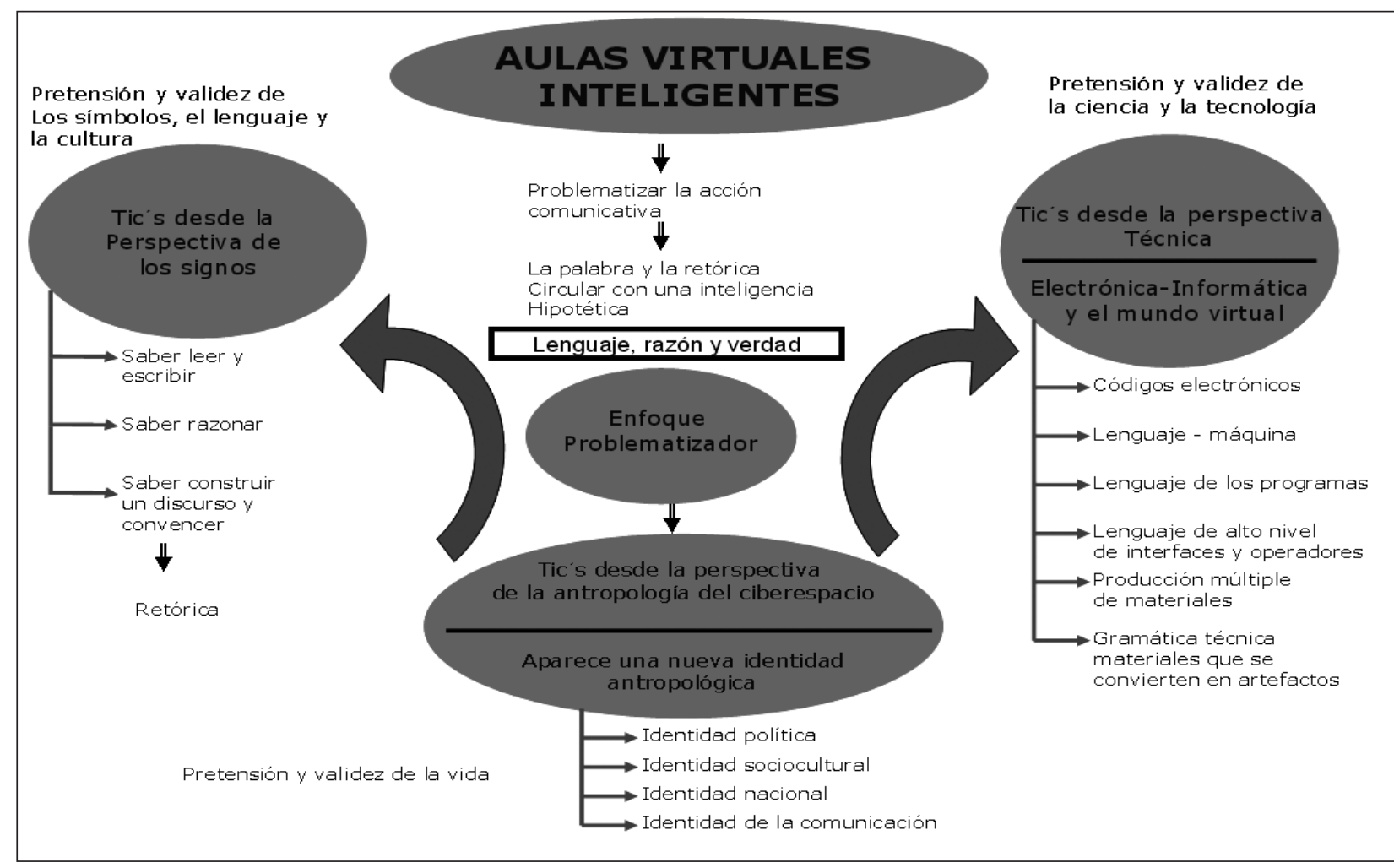

Figura 1.

La educación virtual se debe concebir como un organismo inteligente a partir de un enfoque problematizador de la pregunta y la respuesta. Esto significa concebir la pregunta y la respuesta como un diálogo honesto y sincero que retoma el mundo intersubjetivo, el mundo social y el mundo objetivo. El hecho de concebir un aula virtual inteligente consiste en partir del significado de la práctica interactiva del aprendiente de la vida cotidiana, tomando la lectura y la escritura que realiza del entorno, la cual proporciona elementos para pensar y razonar sobre aquello que brinda una intencionalidad acerca de algo que sucede en el mundo cotidiano, al tiempo que implica construir una biografía que exprese los sentimientos, los afectos, las emociones y los propios intereses para construir un discurso que exprese lo que siente el aprendiente. Por otra parte, el diálogo conlleva a conocer una antropología sobre la identidad social, política, económica, cultural, ética y moral de un grupo o de una sociedad a la cual pertenece el aprendiente. En otros términos, cómo lograr que el aprendiente construya una antropología sobre su propia región, país o continente, implicándose mediante la construcción de la biografía personal acorde con los tópicos temáticos del plan de estudio, ¿cómo lograr que el aprendiente se interese por los problemas ambientales y brinde una 
respuesta ecológica y ambiental a favor de la conservación de la naturaleza?

El aula virtual inteligente con enfoque problematizador le debe permitir al aprendiente dominar la gramática técnica y las herramientas de las nuevas tecnologías para convertirlos en artefactos y en objetos de aprendizaje. ¿Cuál es el significado de la técnica informática para recrear y contemplar un aprendizaje del mundo subjetivo, del mundo social y del mundo objetivo? El aprendiente debe conocer el lenguaje de la máquina, el lenguaje de los programas, el lenguaje de alto nivel de las interfaces y operadores y la producción de múltiples artefactos tecnológicos, el cual involucrará el juego del lenguaje semántico, social y académico, partiendo de indeterminaciones a determinaciones tópicas asertivas, expresando actos cognitivos y morales con uso apropiado de los artefactos tecnológicos.

La técnica posibilitará que el aprendiente encuentre en las proposiciones gramaticales virtuales, en la imagen y en el sonido un espacio para compartir la pretensión de validez argumentativa sobre conceptos correctos con respecto al valor de verdad concedida a las cosas, a las correcciones y a los actos regulativos para llegar a la verdad. Esto quiere decir que las aulas virtuales con enfoque problematizador facilita aprender a aprender los significados relacionados con los signos, con las cosas, con los seres y la técnica para ir a la búsqueda de la verdad, susceptible de un examen cognitivo, metacognitivo, socio cultural, axiológico y contextual.

\section{Conclusión}

Una verdadera aula virtual inteligente debe contemplar la pregunta y la respuesta, por cuanto debe agotar el horizonte de la respuesta mediante la formulación de una serie de preguntas que conduzcan a tocar con la mano el horizonte intelectual, moral y científico. Igualmente, la pregunta y la respuesta deben favorecer el desarrollo de las sensaciones y del lenguaje, persiguiendo no sólo un aula virtual inteligente, sino también los aprendientes se deben volver personas inteligentes con un alto sentido de humanidad y de piedad hacia el otro. Es decir, el aula virtual inteligente no se puede constituir en un sistema instrumental de cálculos y fines, que contribuya al desarrollo cognitivo y a la aplicación de una tecnología sofisticada que convierta al hombre en una persona egoísta y depredadora de la humanidad, como también del medio ambiente. El aula virtual inteligente, de cara al siglo XXI deberá poseer un sello de moralidad, cuando logre del aprendiente un compromiso social y que éste deje de ser un espectador de los problemas del mundo. Entonces, el aula virtual inteligente es aquélla que promueve la formación integral del aprendiente en medio de un afán desmedido por formar parte de la sociedad del conocimiento.

\section{Referencias}

Fabra, P. (2008). Lenguaje, razón y verdad. Madrid: Marcial Pons. 
Heidegger, M. (2007). El ser y el tiempo. México: Fondo de Cultura Económica.

Lévy, P. (1999). ¿Qué es lo virtual? Barcelona: Ediciones Paidós. 